\title{
Perfil psicopatológico de atendimentos em serviço de saúde mental do entorno do Distrito Federal
}

RESUMO | Objetivo: Realizar levantamento epidemiológico das psicopatologias mais frequentemente atendidas em um Centro de Atenção Psicossocial (CAPS) II no município do entorno do Distrito Federal. Método: Estudo transversal descritivo com coleta de dados por análise documental realizada com 2848 registros de atendimento de um CAPS II do município de Valparaíso de Goiás. Resultados: O maior número de atendimentos foi relacionado transtorno de pânico (20,5\%). Os menores números de atendimento foram para o episódio depressivo moderado (1,8\%). Conclusão: Com relação à cobertura de contingente populacional, a atenção em saúde mental da região estudada é adequada. Em relação ao seu perfil, atendimentos a pacientes com transtorno de pânico foram predominantes. Conhecer o perfil epidemiológico dos atendimentos permite melhor direcionar o cuidado, mas é preciso também traçar estratégias que visem à prevenção do adoecimento quando possível e a promoção da saúde mental.

Palavras-chaves: Serviços de saúde mental; Saúde mental; Psicopatologia.

ABSTRACT | Objective: To conduct an epidemiological survey of the psychopathologies most frequently attended at a Psychosocial Care Center (CAPS) II in the city around the Federal District. Method: Descriptive cross-sectional study with data collection by document analysis performed with 2848 records of attendance of a CAPS II of the city of Valparaíso de Goiás. Results: The largest number of visits was related to panic disorder (20.5\%). The lowest attendance numbers were for the moderate depressive episode (1.8\%). Conclusion: With regard to population contingent coverage, mental health care in the studied region is adequate. Regarding their profile, attendance to patients with panic disorder was predominant. Knowing the epidemiological profile of care allows better directing care, but it is also necessary to outline strategies aimed at preventing illness when possible and promoting mental health.

Keywords: Mental health services; Mental health; Psychopathology.

RESUMEN | Objetivo: Realizar una encuesta epidemiológica de las psicopatologías más frecuentemente atendidas en un Centro de Atención Psicosocial (CAPS) II en la ciudad alrededor del Distrito Federal. Método: Estudio transversal descriptivo con recolección de datos mediante análisis documental realizado con 2848 registros de asistencia de un CAPS II de la ciudad de Valparaíso de Goiás. Resultados: El mayor número de visitas estuvo relacionado con el trastorno de pánico (20.5\%). Los números de asistencia más bajos fueron para el episodio depresivo moderado (1.8\%). Conclusión: Con respecto a la cobertura contingente de la población, la atención de salud mental en la región estudiada es adecuada. En cuanto a su perfil, predominó la asistencia a pacientes con trastorno de pánico. Conocer el perfil epidemiológico de la atención permite dirigir mejor la atención, pero también es necesario delinear estrategias destinadas a prevenir enfermedades cuando sea posible y promover la salud mental.

Palabras claves: Servicios de salud mental; Salud mental; Psicopatología.

\section{Iel Marciano de Moraes Filho}

Enfermeiro. Mestre em Ciências Ambientais e Saúde (PUC-GO). Professor do Curso de Enfermagem da UNIP, Campus Brasília. Brasília -DF- Brasil.

\section{Cintia Taumaturgo Fernandes de Negreiros}

Enfermeira. Especialista em Urgência e emergência. Docente da Facesa cursos técnicos. Valparaiso de Goiás - GO- Brasil.

\section{Sarah Rebecca Sousa da Silva}

Enfermeira. Especialista em Urgência e emergência. Docente da Facesa cursos técnicos. Valparaiso de Goiás - GO- Brasil.

\section{Marcio Fernando Rodrigues}

Enfermeira. Especialista em Urgência e emergência.

\section{Recebido em: 22/10/2019}

Aprovado em: 13/12/2019

\section{Mayara Cândida Pereira}

Enfermeira. Mestre em Gerontologia. Coordenadora do Curso de Enfermagem da Universidade Paulista (UNIP) campus Brasília. Brasília -DF- Brasil.

\section{Thais Vilela de Sousa}

Enfermeira. Mestranda em Enfermagem pelo Programa de Pós-Graduação em Enfermagem da Faculdade de Enfermagem da Universidade Federal de Goiás (UFG), Goiânia - GO- Brasil.

\section{Danila Cárita da Silva}

Enfermeira. Especialista Auditoria em Sistemas de Saúde. Docente do curso de enfermagem do Centro Universitário de Goiatuba (UniCERRADO). Goiatuba - GO -Brasil.

\section{Keila Cristina Félis}

Enfermeira. Mestre em Ciências Ambientais e saúde PUC- GO. Docente do curso de enfermagem do Centro Universitário de Goiatuba (UniCERRADO). Goiatuba - GO -Brasil.

\section{Francidalma Soares Carvalho Filha}

Enfermeira. Doutora em Saúde. Docente da Universidade Estadual do Maranhão (UEMA) e do Centro Universitário de Ciências de Tecnologia do Maranhão (UniFacema). Balsas - MA- Brasil.

\section{INTRODUÇÃO}

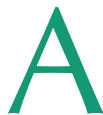
Reforma Psiquiátrica Brasileira, fundada no final dos anos 70 e impulsionada pelo movimento sanitário, condicionou mudanças nos modelos de atenção e gestão das práticas de saúde ${ }^{1}$. Mudanças que superaram a violência asilar e manicomial trazendo à tona os direitos dos pacientes, em plena crise do modelo de assistência centrado no hospital psiquiátrico. Esse processo político e social complexo, composto por vários atores, instituições e forças de diferentes origens, concebeu um conjunto de trans- 
formações de práticas, com saberes, valores culturais e sociais que reconhecidamente forjaram a Política Nacional de Saúde Mental' ${ }^{1}$.

A Política Nacional de Saúde mental constitui-se na redução progressiva de leitos psiquiátricos e também na ampliação e fortalecimento da Rede de Atenção Psicossocial (RAPS), composta pela atenção básica, atenção psicossocial estratégica, atenção de urgência e emergência, atenção residencial de caráter transitório, atenção hospitalar, estratégias de desinstitucionalização e estratégias de reabilitação psicossocial $^{2}$. Especificamente a atenção psicossocial estratégica é operacionalizada pelos Centros de Atenção Psicossocial (CAPS) nas suas diferentes modalidades² ${ }^{2}$.

Os CAPS se responsabilizam pela organização em rede no que se refere a serviços de saúde mental em determinada região territorial. Um de seus objetivos é estabelecimento da comunicação ativa com as demais instituições que compõem a RAPS além de atender ou subsidiar o atendimento a pacientes com sofrimento psíquico leve, moderado ou grave. Desta forma essas unidades realizam o atendimento clínico individual, especializado e familiar com o objetivo central de reinserção social, compreensão do processo psicopatológico de saúde e doença, reestabelecimento do convívio do usuário com a família que muitas vezes se encontra rompido ou fragilizado, ajudando-o na reafirmação dos seus direitos civis e na reinserção do mercado de trabalho e comunidade ${ }^{3-4}$.

O pressuposto biopsicossocial entende que a manifestação dos sintomas psicopatológicos pode ser multideterminada, portanto, ainda não há consenso científico a respeito do surgimento e das causas específicas das grandes síndromes psicopatológicas $^{5}$. Quanto a sua categorização, são consideradas psicopatologias mentais todas as manifestações comportamentais que apresentem alterações de consciência, da atenção, sensopercepção, linguagem, memória, pensamento, volição, psicomotricidade, afetividade e consciência do eu ${ }^{5}$.
Em sua maioria as patologias mentais são marcadas por episódios de alta gravidade clínica e apresentam um manejo complexo tanto para o diagnóstico como prognóstico ${ }^{6}$. Contudo, deve-se entender o conjunto de manifestações como doença e os sintomas de ordem somática que se apresentam nas situações de descontrole emocional, além dos padrões comportamentais que diferem dos padrões sociais impostos e que devem ser acolhidos e tratados de maneira específica como quaisquer outras moléstias ${ }^{7}$.

Atualmente os sistemas classificatórios dos transtornos mentais não apresentaram grandes mudanças quanto sua categorização nosológica, mas oferecem a fidedignidade do diagnóstico com o desenvolvimento de uma linguagem padronizada $^{7}$. Isto ocorre ao se afirmar que um paciente sofre de transtorno bipolar do humor de acordo com a CID-10, classificação é entendida por todos os psiquiatras do continente, mesmo com discordâncias discordem dos critérios ou do mesmo do diagnóstico ${ }^{7}$.

No Brasil, há poucos estudos que relatam o cotidiano de atendimento das unidades de saúde e de transtornos mentais, por isso ressalta-se a importância desta pesquisa, cujo objetivo foi realizar levantamento epidemiológico das psicopatologias mais frequentemente atendidas num Centro de
Atenção Psicossocial (CAPS) II no município do entorno do Distrito Federal.

\section{MÉTODO}

Trata-se de um estudo transversal descritivo realizado no CAPS II de um município no entorno do Distrito Federal. Foram coletadas informações de 2848 atendimentos realizadas no CAPS II no período de janeiro de 2018 a janeiro de 2019. Foram utilizados dados do sistema de informação da Secretaria de Saúde do município, considerando a Classificação Internacional de Doenças (CID) 10. A coleta de dados foi realizada por meio de pesquisa documental em registros de atendimento do CAPS II. Os dados foram organizados em tabelas utilizando o programa Excel do pacote Office versão 2018 e para tratamento estatístico utilizou-se do o pacote Sisvar 5.6. A pesquisa se tratou de um estudo documental em que se utilizou de dados secundários. As variáveis utilizadas na pesquisa foram: Número de atendimentos e diagnósticos psicopatológicos atendidos pelo CAPS II da região.

\section{RESULTADOS}

Os resultados obtidos por meio dos registros foram 2848 atendimentos em um ano demonstrados abaixo.
Gráfico 1. Distribuição dos atendimentos das psicopatologias, no período de janeiro de 2018 a janeiro de 2019, no CAPS II do Município do entorno do Distrito Federal-DF. Brasil, 2019.

\section{Total dos registros de atendimentos do CAPS II}

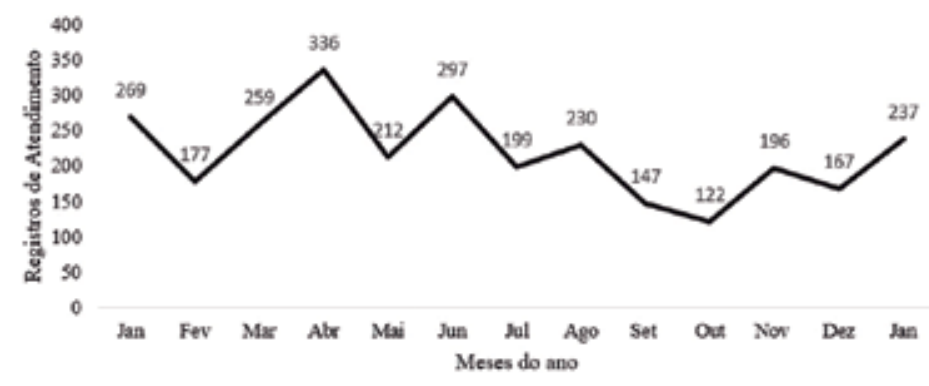


O mês com maior número de atendimentos foi abril e o menor foi outubro. Segundo a análise, o maior número de atendimentos foi com transtorno de pânico e o menor para o episódio depressivo moderado que ocorreu em $1,8 \%$ dos atendimentos.

\section{Gráfico 2. Distribuição dos principais atendimentos, no período de janeiro/2018 a janeiro/2019, no local CAPS II do Municipio do entorno do Distrito Federa-DF. Brasil, 2019.}

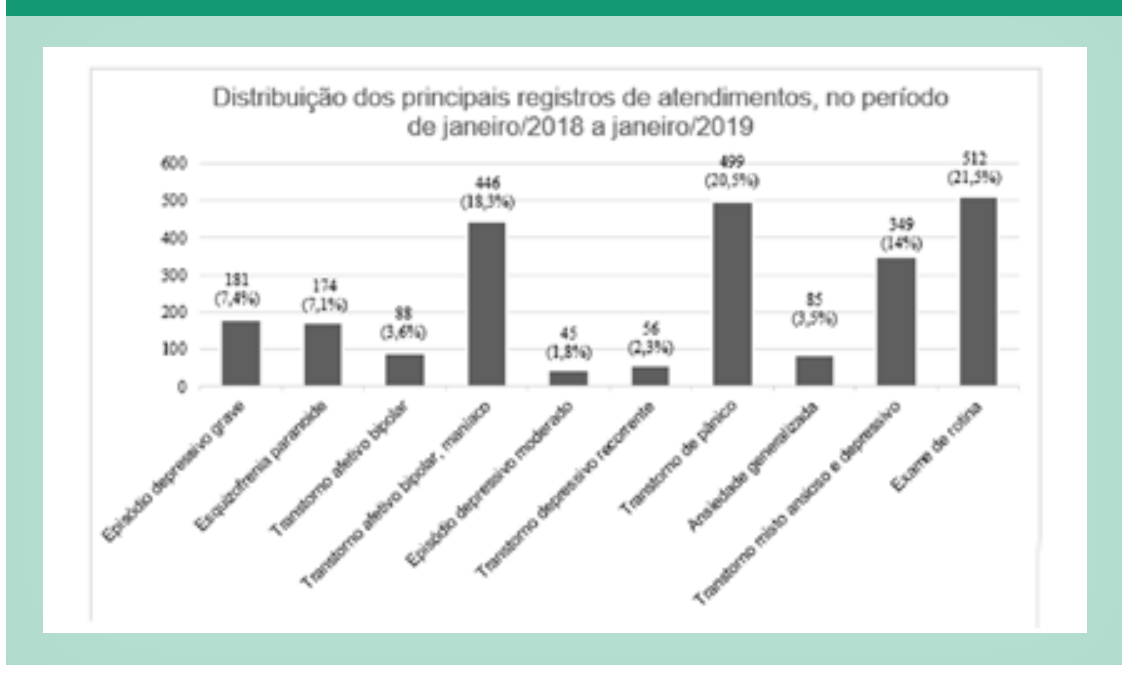

Adicionalmente, em relação ao total de consultas dos principais casos no CAPS II, de janeiro de 2018 a janeiro de 2019, os maiores números referem-se aos exames de rotina, representando 21,5 $\%$. Esses atendimentos são direcionados a solicitação de exames periódicos para avaliação de toxicidade de medicações psicotrópicas para manutenção ou interrupção da conduta medicamentosa e para uma terapêutica segura. Como não se trata de uma psicopatologia, apesar de este dado estar quantificado, não será discutido por não ser foco deste estudo.

\section{DISCUSSÃO}

De acordo com a Organização Mundial de Saúde, aproximadamente 700 miIhões de pessoas possuem alguma psicopatologia de caráter mental que pode ser tanto de cunho neurológico quanto comportamental, levando estes indivíduos ao sofrimento particular ou constrangimento perante a sociedade onde vivem. As psi- copatologias mentais correspondem a $13 \%$ da totalidade de enfermidades mundiais e representam um terço das doenças não transmissíveis ${ }^{8}$.

De acordo com o Instituto Brasileiro de Geografia e Estatística a densidade

demográfica do município pesquisado, localizado no entorno de Brasília, é de $2.165,48 \mathrm{hab} / \mathrm{km}^{2}$. A população estimada para o ano de 2018 era de 164.723 habitantes ${ }^{9}$. Paralelamente a isso, de acordo com a análise dos resultados obtidos e a estimativa populacional através dos dados do IBGE para 2018, supõem-se que $1,72 \%$ da população total do município possuem algum tipo de psicopatologia e necessitou de atendimento de um serviço de saúde por algum processo de adoecimento mental instalado ${ }^{9}$.

Ressaltamos que no município de Valparaíso de Goiás, no entorno do DF, há apenas um CAPS II, compatível com a densidade populacional e legislação vigente pautada na Portaria n. ${ }^{\circ} 336 / G M$ de 19 de fevereiro de 2002, a qual estabelece que municípios com mais de 70.000 a 200.000 habitantes - CAPS II, CAPS AD e rede básica com ações de saúde mental ${ }^{10}$.

$\mathrm{O}$ presente estudo evidenciou o maior número de atendimentos foram à transtorno afetivo bipolar, episódio atual maníaco com sintomas psicóticos, transtorno de pânico, e transtorno misto ansioso e depressivo, totalizando 2848 atendimentos. Estudo semelhante realizado no município de Candeias no estado da Bahia foram analisados 71 prontuários de usuários submetidos ao CAPS II da região e os transtornos mais incidentes, de acordo com o CID-10, foram esquizofrenia, transtornos esquizotípicos e delirante ${ }^{11}$.

Outro estudo realizado no município de Jaguaré no estado do Espírito Santo identificou a maior prevalência nos transtornos de episódio depressivo, misto ansioso e depressivo, afetivo bipolar, esquizofrenia paranoide, retardo mental leve e os transtornos hipercinéticos ${ }^{12}$.

Os registros de atendimento no CAPS II do município observado nesta pesquisa totalizaram 2435 atendimentos. Estudo realizado no Estado de Minas Gerais, no ano de 2018, na cidade de Paraopeba no estado de Minas Gerais com um quantitativo de 21 mil habitantes, demostrou que a número de atendimentos menor em comparação ao estudo referido o qual registrou entorno de 3 mil pelos centros observados. Nesse ainda estava incluindo consultas programadas e de urgência atendidas pela equipe multiprofissional ${ }^{13}$.

Em outro estudo no estado de Rondônia, teve 2.383 consultas no período de 12 meses do ano de 2014 semelhante com os resultados encontrados no município pesquisado ${ }^{14}$.

A OMS preconiza a necessidade de se criar estratégias para reduzir os efeitos acometidos pelos transtornos de caráter mental mundialmente e aperfeiçoar as competências e habilidades dos profissionais que atendem diretamente os usuários, objetivando o defronte, evitando assim a intensificação dele $\mathrm{e}^{15}$.

Os atendimentos dos usuários do CAPS podem ser entendidos como um comportamento que diz respeito a conduta do profissional ao recepcionar, ouvir e atuar de forma humanizada no acolhimento do paciente de acordo com a sua necessidade. É importante que o profissional use de estratégias e atitudes de modo 
organizado, possibilitando o entendimento da complexidade das queixas e sofrimentos. Deve estar preparado para ouvir e acolher, mas investigar, analisar e ter discernimento para gerenciar riscos da demanda e necessidades, procurando solucioná-las de modo holístico favorecendo a sua singularidade biopsicossocial ${ }^{15-16}$.

Os maiores registro de atendimento referem-se ao transtorno de pânico com 20,5\% dos atendimentos anuais. Em relação aos menores números de registros, houve episódio depressivo grave com 1,8\%. Em pesquisa realizada no estado de São Paulo na região metropolitana em relação aos últimos 12 meses, houve 29,6\% de transtorno generalizado de ansiedade $(19,9 \%)$, os transtornos de humor $(11,0 \%)$ seguidos de transtornos de controle de impulso $(4,2 \%)$ e os decorrentes do uso de substâncias psicoativas (3,6\%)17. Estima-se que na cidade de São Paulo a prevalência da doença mental (sem distinguir os subtipos) por toda sua vivência corresponde a 1\% (3000) e a prevalência anual gira em torno de 5\% (600).

Na cidade de Valparaíso de Goiás, município pesquisado com 164.723 habitantes, a prevalência em relação ao total de habitantes $(1 \%)$, fora apontada para 1.647,23 casos de vivência, e para a prevalência anual, o estudo apontou $8.236,15$ casos anuais (5\%). A pesquisa traz como resultado as psicopatologias prevalentes da região, o transtorno de ansiedade generalizada correspondeu a $3,5 \%$, o afetivo bipolar $3,6 \%$ e o afetivo bipolar maníaco 18,3\%. No presente estudo o episódio depressivo moderado correspondeu a $1,8 \%$ da amostra estudada, seguido pelo transtorno depressivo recorrente que foi $2,3 \%{ }^{9}$. Caracteriza-se por distúrbio depressivo recorrente as repetidas crises de depressão, com humor abatido, e o indivíduo sente-se desinteressado, logo ele não sentira entusiasmo nas tarefas habituais por um período de, no mínimo, quinze dias 5 .

Outro fator preponderante são quando os episódios depressivos podem ser de categorias diferentes, leve, moderada ou grave, isso dependerá da expressividade dos sintomas clínicos do paciente. Os episódios leves causam dificuldades nas atividades cotidianas, mas não são fatores determinantes para um agravamento geral

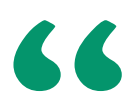

\section{transtorno afetivo bipolar identificou- se como a terceira ocorrência de maior incidência em estudo realizado numa unidade de CAPS} I, representando $10 \%$ do total de diagnósticos, com predominância no sexo feminino em mais da metade dos diagnósticos $^{12}$.

do usuário, ao contrário do episódio grave, que impossibilitará o indivíduo acometido de realizar suas funções comuns corriqueiras $^{5}$. Ambos os tipos de depres- são podem ser crônicos e apresentarem momentos de reincidências. A OMS descreve que a depressão está em ascendência no mundo, devido a preocupação com a evolução da doença isso fez com que uma resolução aprovada em maio de 2013 exigisse nacionalmente respostas globais em modos coordenados ${ }^{12}$.

Com relação ao transtorno misto ansioso e depressivo, na população investigada o índice foi $14 \%$ dos atendimentos, dados semelhantes foram encontrados em um estudo realizado na região do estado de Espírito Santo, o qual identificou que foi identificado como a segunda maior ocorrência de incidência e a parcela representada foi cerca de $14 \%$ das patologias diagnosticadas ${ }^{12}$. O transtorno apresenta sintomas depressivos e maníacos que podem se alternar ou se apresentarem ou concomitantemente, além de ser de difícil diagnóstico ${ }^{5}$.

O transtorno afetivo bipolar identificou-se como a terceira ocorrência de maior incidência em estudo realizado numa unidade de CAPS I, representando $10 \%$ do total de diagnósticos, com predominância no sexo feminino em mais da metade dos diagnósticos ${ }^{12}$. De acordo com a OMS são reconhecidos vários motivos para a maior prevalência de transtornos de depressão e ansiedade, tais como fatores genéticos, biológicos e socioculturais ${ }^{12}$.

A esquizofrenia paranoide mostrou-se em outro estudo, com $68 \%$ dos casos, como a psicopatologia em quarto lugar na prevalência dos atendimentos realizados. A prevalência foi maior no sexo masculino e nos mais jovens ${ }^{12}$. Os sintomas são delírios, que por vezes são paranoides, alucinações de percepção e alucinações auditivas. De acordo com a OMS este é o tipo de esquizofrenia que mais acomete os indivíduos na maioria dos paíse ${ }^{\text {s12. }}$.

Nesta investigação, verificamos que 3,5\% da população possuem ansiedade generalizada. Vinte e três estudos epidemiológicos mostraram que os transtornos de ansiedade generalizada têm variação entre $0,7 \%$ e $24 \%$ e a prevalência destes não chegaram a 3\% em 43,48\% dos estu- 
dos avaliados. Dentre os estudos, apenas 4 foram realizados no brasil, mostrando que os resultados variaram por se tratarem de regiões com diferenças nas características sociodemográficas e no quantitativo das amostras. Este transtorno pode apresentar-se em fases diferentes ao longo da vida e em cada uma delas as características são particulares e apresentam-se junto a outras patologias mais agressivas ${ }^{5}$. Por se ratar de dados consideráveis de prevalência, pode ser estimado como um problema de saúde pública, por trazer consequências à saúde e por se tratar de um diagnóstico negligenciado nos níveis de atenção ${ }^{18}$.

Verificou-se também que 20,5\% do total da amostra apresentou quadro de transtorno de pânico. A OMS e o CID 10 reconhecem o transtorno de pânico e o aloca na classe dos transtornos mentais ${ }^{5}$. O ataque de pânico é descrito pela presença de ataques recorrentes baseados em sensação de fobia ou mal-estar excessivo junto a sintomas físicos e cognitivos e que se inicia de modo súbito atingindo o ápice máximo em 10 minutos $^{19}$.

Devido a prevalência dos ataques de pânico na população em geral e a necessidade de se saber diferenciá-los em seus diversos aspectos, o conhecimento dos profissionais se torna imprescindível para o desnovelar da caracterização do diagnóstico e logo iniciar o plano terapêutico que é de extrema importância para a meIhora efetiva do usuário ${ }^{17,20}$.

\section{CONCLUSÃO}

Diante a análise sobre o levantamen- to epidemiológica conduzido e considerando o município de realização da pesquisa é possível concluir que, em relação à cobertura de contingente populacional, a atenção em saúde mental da região estudada é adequada. Quanto ao conhecimento de seu perfil, em que predominou atendimentos a pacientes com transtorno de pânico, possibilita o fomento de novas estratégias que visem à melhoria, atendendo demandas biopsicossociais e personalizando o cuidado de acordo com esse perfil, proporcionado assim esperança de vida com qualidade aos indivíduos acometidos por essa doença mental. Além disso, de uma forma geral, é preciso também traçar estratégias e criar programas efetivos que visem à prevenção do adoecimento quando possível e a promoção da saúde mental.

\section{Referências}

1. Ministério da Saúde. Reforma psiquiátrica e política de saúde mental no Brasil. Conferência Regional de Reforma dos Serviços de Saúde Mental: 15 anos depois de Caracas. Brasília: Ministério da Saúde; 2005. 2. Ministério da Saúde; Secretaria de Atenção à Saúde; Departamento de Ações Programáticas Estratégicas. Saúde mental no SUS - Cuidado em liberdade, defesa de direitos e rede de atenção psicossocial. Relatório de gestão 2011-2015. Brasília: Ministério da Saúde; 2016.

3. Moraes Filho IM, Carvalho Filha FSS, Almeida RJ. Serviços de atendimento públicos para usuários de álcool e outras drogas. Rev Cient Sena Aires. 2016; 5(1):1-3.

4. Leal BM, Antoni CD. Os Centros de Atenção Psicossocial (CAPS): estruturação, interdisciplinaridade e intersetorialidade. Aletheia. 2013;(40):87-101.

5. Dalgalarrondo P. Psicopatologia e semiologia dos transtornos mentais. Porto Alegre: Artmed; 2018

6. Drummond BLC, Radicchi ALA, Gontijo ECD. Fatores associados a transtornos mentais com situações de risco na atenção primária de saúde. Rev Bras Epidemiol. 2014; Suppl (D.S.S): 68-80.

7. Barlow D, Durand V. Psicopatologia: uma abordagem integrada. São Paulo: Cengage leaening, 2008.

8. Valença AM, Nascimento I, Mecler K, Freire R, Mezzasalma MA, Leão $\mathrm{V}$, et al. Comportamento violento, gênero e psicopatologia. Rev Latinoam psicopatol fundam. 2018;13(2):1-15.

9. Instituto Brasileiro de Geografia e Estatística. Número total da população do Município de Valparaíso de Goiás. IBGE; 2019.

10. Ministério da Saúde. Portaria GM/MS n 336 , de 19 de fevereiro de 2002. Estabelece que os Centros de Atenção Psicossocial poderão constituir-se nas seguintes modalidades de serviços: CAPS I, CAPS II e CAPS III, definidos por ordem crescente de porte/complexidade e abrangência populacional. Brasília: Ministério da Saúde; 2002.

11. Cruz LS, Carmo DC, Sacramento OS, Almeida MSP, Silveira HF, Ribeiro Junior HL. Perfil de Pacientes com Transtornos Mentais atendidos no Centro de Atenção Psicossocial do Município de Candeias - BA. Rev
Bras Cienc Saúde. 2016; 20(2):93-98.

12. Leitão IB, Figueiredo DD, Marbach MAJ, Martins KS. Caracterização dos transtornos psiquiátricos diagnosticados no CAPS I, em Jaguaré, ES, no período de janeiro a outubro de 2014. Rev Psicol Saúde. 2017;9(1):19-35.

13. Silva SN, Lima MG. Assistência Farmacêutica na Saúde Mental: um diagnóstico dos Centros de Atenção Psicossocial. Rev Ciência \& Saúde Coletiva. 2017; 22(6):2025-36.

14. Franskoviak LD, Silva TE, Carlotto, MS, Batista EC. Perfil epidemiológico de usuários de psicotrópicos de um CAPS da Zona da Mata do Estado de Rondônia. Rev Interdiscip Estud Saúde. 2018;7(1):68-82.

15. Scheibel A, Ferreira LH. Acolhimento no CAPS: reflexões acerca da assistência em saúde mental. Rev Baiana Saúde Púb. 2011;35(4): 966 983.

16. Moraes Filho IM, Santos OP, Félis KC, Caetano SRS. Concepções de enfermeiros de um centro de atenção psicossocial sobre o cuidar a adultos com transtornos mentais graves- relato de experiência. Revisa. 2015; 4(2): 86-95.

17. BonadimanI CSC, Passos VMA, Mooney M, Naghavi M, Melo APS. A carga dos transtornos mentais e decorrentes do uso de substâncias psicoativas no Brasil: Estudo de Carga Global de Doença, 1990 e 2015. Rev Bras Epidemiol. 2017;20(Suppl 01):191-204.

18. Menezes AKS, Moura LF, Mafra VR. Transtorno de ansiedade generalizada: uma revisão da literatura e dados epidemiológicos. Rev Amazônia Science \& Health.2017;5(3):42-49.

19. Pinto DM, Jorge MSB, Pinto AGA, Vasconcelos MGF, Cavalcante CM, Flores AZT, et al. Projeto terapêutico singular na produção do cuidado integral: uma construção coletiva. Texto contexto - enferm. 2011;20(3): 493-502.

20. Moraes Filho IM, Moura LM, Souza AM, Pereira LS, Santos LCN, Arantes $A A$, et al. $A$ atuação da enfermagem na oficina terapêutica no contexto de um centro de atenção psicossocial. REAEnf/EJNC. 2019;1(e1452): 1-7. 